\title{
Gender Disparity and Socio-Demographic Characteristics of Adolescents in Northern Nigeria
}

By

\author{
${ }^{1}$ Godswill James, $\mathrm{PhD}$ and ${ }^{1} \mathrm{James}$ Amos Akpokos
}

\begin{abstract}
This paper gives insights into the adolescents' socio-economic and demographic compositions and brings to limelight the various issues that affect their wellbeing. It also provides available data that can be translated into meaningful information which can be used to establish guidelines for the development of appropriate policies and programmes for the welfare of adolescents. The present study was conducted in three states in NorthWestern Zone of Nigeria, namely Kaduna, Kano and Katsina. These states have the highest total percentage of teenagers who have begun child bearing in the North-West zone, more than half of the total projected population of the zone and about $60 \%$ of the total projected population of teenagers age 12-19 in the zone. Questions relating to use of methods of contraception were also asked. Data on knowledge and use of contraceptive methods are central to demographic analysis particularly fertility phenomenon. This study highlighted the immediate need for a concerted effort towards solving the several problems confronting the adolescents in northern Nigeria.
\end{abstract}

Key Words: Gender, children, teenager, disparity, knowledge, contraceptive methods

\section{Introduction}

Children, adolescents and youth constitute a large proportion of Nigeria's population. They have special needs throughout their life cycle, and several international conferences and summits, which have focused on children and young people, have emphasized the importance of addressing these needs. These include the Convention on the Rights of the Child (CRC, 1989); the World Summit for Children (1990); the International Conference on Population and Development (ICPD, 1994); the Beijing Platform for Action (1995); the UN General Assembly Special Session on HIV/AIDS (2001); and UN General Assembly Special Session on Children (2001). Nigeria is a signatory to both the United Nations Convention on the Rights of the Child and the African Union (AU) Charter on the Rights and Welfare of the Child. This has placed the

${ }^{1}$ Department of Sociology, Kaduna State University, Kaduna, Nigeria 
issue of human rights, especially the rights of children, youth and women, at the forefront of national concern.

Particular concerns and needs related to children and young people in Nigeria include the eradication of preventable diseases like polio, tuberculosis, and diphtheria; improved levels of educational attainment (especially formal education of the girl child); rights to reproductive and sexual health; HIV/AIDS; prevention of maternal deaths and disabilities; and increased employment opportunities or job creation. Essentially, the progress of children and young people has become a key goal of overall national development. Consequently, it has become very necessary to improve the opportunities open to young people so that they will grow their fullest potential and have the solid foundation to enable them take their rightful places as future leaders.

Strong commitment to the development of, and investment in children, adolescents and youth is a cornerstone to people-centred sustainable development; they constitute not only a formidable demographic force, but also make up the next generation of parents, labour force and leaders. Their wellbeing, therefore, has ramifications not only for their own lives, but also for the lives of children they bring into the world, and for the societies they will build and maintain. Meeting their needs is a major continuing public policy challenge which calls for constant re-thinking of policies, re-assessment of priorities, and commitment of adequate financial resources and effective implementation of programmes. More efficient and equitable resource allocation and improved policy formulation and implementation can only be achieved with a better understanding of their needs. An essential prerequisite for such an understanding is the availability of timely, reliable and comprehensive data on their characteristics (NPC, 2002).

This research report on adolescents gives insights into their socio-economic and demographic compositions and brings to limelight the various issues that affect their wellbeing. It also provides available data that can be translated into meaningful information which can be used to establish guidelines for the development of appropriate policies and programmes for the welfare of adolescents.

\section{Conceptual Framework}

The term "adolescent" is variously defined as "the state or process of growing up", "the period of life from puberty to maturity", and "the period of transition from childhood to adulthood, encompassing both the development to sexual maturity, and to psychological and relative economic independence" (United Nations, 1988a; IPPF, 1994:5). There is no precise and consistent age range that defines when adolescent begins and ends in adolescence studies; operational definitions vary depending on culture and place. United Nations $(1987,1988$ a,1989), for instance, chose to look at the reproductive behavior of teenagers, that is, those in the age range 13-19, in its work on this subject. Other studies focus on the age range 15-19 (Bledsoe and Cohen, 1993; Population Reference Bureau, 1992; 2005; Yinger et al., 1992; World Health Organization, 1989a, 1989b). However, studies of "youth" tend to address a broader and somewhat older age group, that is, young people, ages $15-24$. As a practical matter, most teenage reproductive behavior tends to occur in the age range 15-19 in most populations. However in Nigeria, 
particularly the northern part, childbearing starts much early. Therefore for the purpose of this study, the term "adolescent" refers to young people ages 12-19, which is an approximation of the post-puberty population under age 20 . Throughout this paper, the terms "adolescent" and "teenager" are used interchangeably.

\section{Materials and Methods}

The present study was conducted in three states in North-Western Zone of Nigeria, namely Kaduna, Kano and Katsina. These states have the highest total percentage of teenagers who have begun child bearing in the North-West zone, more than half of the total projected population of the zone and about sixty percent of the total projected population of teenagers age 12-19 in the zone.

A multi-stage sampling method was used in this research. In each of these states, two urban and two predominantly rural Local Government Areas (LGAs) were selected, one each from North and South of the states using simple random sampling techniques, after all the LGAs in each of the selected states were listed according to their setting (urban or rural) as well as geographical zone (north or south). Thus, six urban towns and six rural villages were selected from the states for detailed study; three were drawn from the southern part and the other three from the northern part of the states. They included the following:

- $\quad$ Kaduna- (i) Giwa, (ii) Kafanchan, (iii) Zango Kataf, and (iv) Zaria City

- $\quad$ Kano- (i) Dala, (ii) Kunchi, (iii) Rimin Gada, and (iv) Tudun Wada

- $\quad$ Katsina- (i) Bindawa, (ii) Daura, (iii) Funtua, and (iv) Matazu

A number of main streets were randomly selected in each town and village, from which houses and households were systematically chosen to make up the sample for a particular area. In each household, one female and/or male adolescent were interviewed. In a household where there were more than one male and one female adolescent, a simple random sampling technique was used to select one male and one female. However, in the case of married or cohabiting adolescents, one male and his female partner were interviewed. In all, 1175 adolescents (606 males and 569 females) were interviewed successfully, using structured questionnaires that elicited information about the respondent's socio-economic and demographic background; respondent's parental background; sexual and reproductive status; family size preferences; knowledge of, attitude toward, and practice of contraceptives, as well as reproductive attitudes and motivations.

In the analysis, simple descriptive data are presented with respect to questions on the respondent's gender, geo-political place of birth, religion, ethnic group, marital status, education level, knowledge of contraception, approval of contraception, use of contraception, paternal and maternal education, paternal and maternal ages of first marriage, paternal and maternal number of living children, and paternal type of marital union. The aim of the analysis is to show how the circumstances of the respondents differ by their gender. 


\section{Results}

\section{Age and Sex Distribution}

The age and sex distribution of any population is a very important demographic parameter and has many significant implications. Throughout this report, 'adolescents' refer to persons of ages 12-19 years. This group embraces junior (JSS) and senior (SSS) secondary school population, as well as some population in the early part of tertiary institution. Persons in this group are distinct group in terms of their health needs. Moreover, issues of reproductive health, including early marriage, teenage pregnancy and childbearing, are critical among this group.

Table 1 shows, among other characteristics the age distribution of sample respondents by sex. The age group adopted in this report was guided by the need to provide data and analysis that have practical, research and policy relevance within Nigeria. For instance, adolescents aged 12-17 years within Nigerian setting, have basic need of secondary education and the acquisition of other life sustaining skills and information. On the other hand, any person who reaches the age of 18 years is legally considered an 'adult' with voting rights. This group of persons would need post secondary education, employment and reproductive health information and services.

Overall, more than $46 \%$ of all respondents were 17 years or less, while about $53 \%$ are 18 years or more. The age distribution of the respondents by sex shows that there were more males $(49.2 \%)$ than females $(43.8 \%)$ among adolescents aged 17 years or less, but more females $(56.2 \%)$ than males $(50.8 \%)$ among adolescents aged 18 years or more. On the whole, there were approximately 120 males for every 100 females among adolescents aged 17 years or less, and 96 males for every 100 females among adolescents aged 18 years or more.

The above data indicate the fact that experiences of adolescents at different age groups during marital and childbearing age are well represented in the data generated in this study.

\subsection{Place of Birth and Ethnic Group of Origin}

The place of birth and ethnic group of origin has salient implications on the reproductive behavior of a child. Ethnicity itself is the basis of social organization in the traditional context and it encompasses a mosaic of observable norms, beliefs and rituals that govern various life events (Gyimah, 2002b). Each ethnic group has its own corpus of knowledge and practices in the sphere of health and childcare. The fact that there are many social groups with distinct cultural traits in Nigeria is reflected in diverse behavior of the people. 
Table 1: Percent distribution of respondents' socio-demographic characteristics by sex

\begin{tabular}{|c|c|c|c|}
\hline Characteristics & $\begin{array}{c}\text { Male } \\
(\mathrm{N}=606)\end{array}$ & $\begin{array}{c}\text { Female } \\
(\mathrm{N}=569)\end{array}$ & $\begin{array}{c}\text { All } \\
(\mathrm{N}=1175)\end{array}$ \\
\hline Age & $\%$ & $\%$ & $\%$ \\
\hline$\leq 15$ years & 11.1 & 10.7 & 10.9 \\
\hline $16-17$ years & 38.8 & 33.1 & 35.7 \\
\hline$\geq 18$ years & 50.8 & 56.2 & 53.4 \\
\hline \multicolumn{4}{|l|}{ Place of birth } \\
\hline North Central & 7.4 & 9.4 & 8.3 \\
\hline North East & 2.3 & 4.5 & 3.4 \\
\hline North West & 80.9 & 77.4 & 79.2 \\
\hline South West & 2.5 & 4.4 & 3.4 \\
\hline South East & 3.6 & 2.0 & 2.8 \\
\hline South South & 3.3 & 2.3 & 2.9 \\
\hline \multicolumn{4}{|l|}{ Ethnic group } \\
\hline Hausa/Fulani & 45.9 & 59.8 & 52.7 \\
\hline Yoruba & 10.6 & 7.9 & 9.3 \\
\hline Igbo & 5.1 & 2.3 & 3.7 \\
\hline Other & 38.4 & 30.0 & 34.3 \\
\hline \multicolumn{4}{|l|}{ Religion } \\
\hline Christianity & 18.2 & 16.7 & 17.5 \\
\hline Muslim & 76.7 & 79.7 & 78.2 \\
\hline Pagan/Traditionalist & 5.1 & 3.6 & 4.3 \\
\hline \multicolumn{4}{|l|}{ Ever attended school? } \\
\hline Yes & 97.9 & 73.1 & 85.9 \\
\hline No & 2.1 & 26.9 & 14.1 \\
\hline \multicolumn{4}{|l|}{ Highest level of education } \\
\hline No formal schooling & 3.5 & 25.0 & 13.9 \\
\hline Some primary & 12.2 & 29.8 & 20.7 \\
\hline Some Secondary & 49.9 & 33.0 & 41.7 \\
\hline Some tertiary & 34.4 & 12.2 & 23.7 \\
\hline \multicolumn{4}{|l|}{ Ability to read and write } \\
\hline Without any difficulty & 78.1 & 47.3 & 63.2 \\
\hline With difficulty & 18.3 & 27.6 & 22.8 \\
\hline Cannot & 3.6 & 25.1 & 14.0 \\
\hline \multicolumn{4}{|l|}{ Marital status } \\
\hline Single & 78.7 & & 67.7 \\
\hline Married & 20.5 & 56.1 & 30.4 \\
\hline Divorced/Separated & 0.8 & 40.9 & 1.2 \\
\hline Widowed & 0.0 & 1.6 & 0.7 \\
\hline Occupation & & 1.4 & \\
\hline Civil/public Servant & 8.9 & 5.3 & 7.1 \\
\hline Business/Petty trading & 21.0 & 20.0 & 20.5 \\
\hline Farming & 7.1 & 9.3 & 8.2 \\
\hline Artisan & 13.9 & 13.7 & 13.8 \\
\hline Student/homemaker/ unemployed & 49.1 & 51.7 & 50.4 \\
\hline
\end{tabular}

(C) 2013 The Authors. Journal Compilation ～(C) 2013 European Center of Sustainable Development. 
The distribution of respondents according to place of birth and ethnic group of origin is presented in Table 1. For ease of analysis the respondents' place of birth was grouped on the basis of the geo-political zones, while their ethnic group of origin was categorized according to the nation's major and minority ethnic groups. Within the boundary of Nigeria, there are many identifiable ethnic groups but Hausa/Fulani, Yoruba and Igbo are the major ones. The 'other' ethnic groups fall under the broadest groupings of linked ethnic unit by region, that is, north and south minority ethnic group (NPC, 2004).

It is evident from the data in Table 1 that the overwhelming majority of both male and female respondents were born in the North West geo-political zone, and slightly more than half of them $(52.7 \%)$ were Hausa/Fulani. This is not surprising in view of the fact that the study was conducted in the North West geo-political zone in which many of the inhabitants are Hausa/Fulani by ethnic origin. The three southern regions had $9.1 \%$ of the total sample size with a glaring disparity in sex distribution which is skewed toward males. This proportion of the study sample represents the off springs of migrant parents from southern states in pursuit of education, employment or business.

\subsection{Religious Affiliation}

Religion is a very important socio-cultural factor in the Nigerian society. It serves as motivation for what people believe and practice and so can be an effective determinant of the society's response to fertility and other behavior. In fact religion is widely accepted as an important predictor of demographic behavior in every human society. It influences cultural and social beliefs of people in every known human society. In addition, it serves as a significant medium by which traditional cultural beliefs and norms can be modified. Through it, foreign attitudes and values are learnt and some biases acquired (Adeokun, 1979).

Table 1 shows the percent distribution of respondents by sex and religious affiliation. About one in every six respondents $(17.5 \%)$ were Christians while $78.2 \%$ were Moslems and the traditionalist/pagan constituted $4.3 \%$. Essentially, Islam and Christianity are two main religious groups in Nigeria; the third is the indigenous traditional religion, which comprises a number of traditional beliefs and practices. The dominance of Muslims in the study area is not unexpected because the survey drew respondents from both urban and peripheral or rural settlements of Kaduna, Kano, and Katsina states which are almost exclusively Muslim settlements.

\subsection{Literacy and Educational Attainment}

Educational attainment is closely linked with other socio economic factors, such as income, lifestyle, reproductive behavior, health status, etc. Demographers have acknowledged that the time devoted to education leads to delay in marriage and the knowledge acquired during the period brings about a change in people's perception about their family size and reproductive behavior. Additionally, educational attainment alienates people from pro-natalist thinking and traditional environment and culture since most of the lucrative job opportunities associated with education are not usually located in the rural areas where the sacredness of traditional institution is still very much intact.

Table 1 shows percent distribution of respondents according to sex, literacy and educational attainment. Respondents were asked whether or not they have ever attended 
school, and the highest level of education attained as well as the ability to read and write with understanding in any language. About $14 \%$ of the respondents never attended school, and female respondents were more likely than their male counterpart not to have ever attended school. Similarly, about one in every five respondents $(20.7 \%)$ had attained at least some primary education only, and $65.4 \%$ of the adolescents had some education beyond the primary level. As expected, the proportion of the adolescents that had attained higher education was low and highly skewed towards the female gender.

Although, a majority of the respondents $(63.2 \%)$ were able to read and write with understanding without any difficulty, more than half (52.7\%) of females were either able to read and write with difficulty or cannot. Overall, males had higher literacy levels than females; males were more likely than females to have ever attended school, less likely to have no formal schooling, more likely to have attained higher education, and more likely to read and write without any difficulty. The difference in literacy rate between males and females is obviously large $(30.8 \%)$, suggesting that some gender imbalances in literacy among adolescents clearly exists in northwestern Nigeria. This reflects the persistent effects of a tradition which dissuades formal schooling because of its perceived "corrupting" influences on females (Wall, 1998), as well as gender preferences and prohibitions.

\subsection{Marital Status}

Marriage in Nigeria, as in many other societies, provides the primary social setting in which the biological event of child bearing occurs. It is one important proximate determinant of fertility. Marriage is universal, and most child births among adolescents, particularly in northern Nigeria, occur within its institution. For this study, marriage was defined as any stable union, irrespective of whether the male partner has performed certain traditional rites or not. Thus both formal and informal unions were taken to mean marriage because of the significant effects both have on childbearing in Nigeria.

Table 1 shows the percent distribution of respondents according to marital status and sex. The data show that $67.7 \%$ of the respondents had never married, $30.4 \%$ were currently married, and $1.9 \%$ were divorced, separated or widowed. This means that over $32 \%$ of all the adolescents in the sample have ever married. Females were almost two times $(40.9 \%)$ more likely to marry than males $(20.5 \%)$, suggesting that teenage marriage was more common among females than males.

Given that the age at which a woman first gets married influences the length of time she is exposed to the risk of pregnancy during her childbearing years, analysis of the age at first marriage among the respondents that reported ever married was attempted. Data indicated that about $3 \%$ of them married at age younger than 10 years, $27.4 \%$ married between 10 and 14 years of age, 40.1\% married at age between $15-17$ years, while $69.7 \%$ married between 15 and 19 years of age.

Analysis of the differences in cumulative percent ever married by age at first marriage showed that in cumulative terms only $6.4 \%$ of males have had a first marriage by age 14 years and $19.3 \%$ by age 17 years. In contrast, $27.8 \%$ of females were married by age 14 years, and $68.2 \%$ married by age 17 years. The median age at first marriage for the sample was 16.1years for females and 18.7 years for males (17.3 years for both sexes). 
The significant differentials in age at first marriage between male adolescents and their female counterparts confirm the earlier observation that females marry earlier than males in northwestern Nigeria. Given the general observation that the North West is the region with the lowest median age at first marriage in Nigeria, and judging from the 2003 Nigeria Demographic and Health Survey (NDHS) which found that the region's age at first marriage among women age 20-24 years was $15.5 y e a r s$, the data from this study suggest that the median age at first marriage in the region is rising.

\subsection{Occupation}

The distribution of respondents by their occupational status is shown in Table 1 . Occupation has probably been one of the widely used indices of socio-economic status in demographic studies, especially in the assessment of economic activities and evaluation of their contributions to fertility and mortality behaviors. Respondents were asked whether or not they were currently working, that is doing something to generate income, and what type of work they were doing.

Slightly less than half $(49.6 \%)$ of the respondents said they were doing something to generate income. About $51 \%$ of males compared to $48 \%$ of females engaged in one economic activity or another. Majority of both males and females engaged in some informal sector employment particularly business/petty trading and artisanship. Only $7.1 \%$ of the respondents most of whom were males, engaged in formal employment. It is possible that most of the adolescents who reported being engaged in business/petty trading or artisan, did so for their parents or on family-owned business enterprise because the culture in which young persons are seen as sources of unpaid labor for parents is still an acceptable practice in northern Nigeria. On the whole, more than half $(50.4 \%)$ of the overall sample most of whom were females, were not doing something to generate income. They were students, homemakers or adolescents seeking employment.

The high level of unemployment and the predominance of informal sector's employment among the respondents are not unusual and invariably tied to the level of education which according to the data in Table 1 show clearly that $54 \%$ of the respondents have lower than completed secondary school education. Moreover, Nigerian economy has been classified by ILO (1986) to be a mixed trading economy, that is, a type of national economy that falls in-between agricultural and industrially influenced economies. Countries in this type of economy are moving out of pure agricultural activities to trading activities but not yet into industrial activities where informal trading and economic activities are booming and flourishing (Bledsoe and Cohen, 1993:123).

It should be noted that the informal sector of the economy is that sector that is not regulated by way of legislation or government policies. It is unofficial and unregulated sub sector of the economy where free entry to new enterprises exists. These enterprises rely on indigenous resources, they are family-owned and small-scale; they use laborintensive and adapted technology; their workers rely on non-formal sources of education and skills and operate in unregulated and competitive markets. This sector according to Vandemoortele (1991) has become the labor sponges in sub-Saharan Africa after the introduction of Structural Adjustment Programme (SAP) in the region. 


\subsection{Respondents' Parental Background}

Given the crucial influence of parents and parental socio-economic characteristics on children's (including adolescents) behavior, life's circumstance and decision making, Table 2 show the distribution of respondents according to some parental socioeconomic background. Some of the parental characteristics that are found to have bearing on children's behavior include socio-economic status of the family, the educational attainment, reproductive history (including age at first marriage, type of union, number of surviving children) and occupation. These parental characteristics are likely to influence children's attitudes as well as decision making with regards to choice of career, age at marriage, type of union and family size.

As expected, parental education was low and mothers tended to have lower level of education than fathers. For instance, respondents' mothers were more likely $(46.2 \%)$ to have no formal education than respondents' fathers (26.3\%). On tertiary education, about $25 \%$ of mothers as against $47 \%$ of fathers had higher education. Overall, $62.3 \%$ of mothers and $37 \%$ of fathers had primary education or lower, and fathers were more likely than mothers to complete each level of education.

Table 2 also shows percent distribution of respondents according to parental occupation. The data show that only $31.2 \%$ of fathers and $19.6 \%$ of mothers were engaged in public sector employments. Majority of fathers were engaged in informal sector employment and $3.8 \%$ of them were unemployed. On the other hand, while some mothers were engaged in informal sector employment, particularly business/petty trading, a large proportion of them (47.6\%) were homemakers and unemployed. This is not surprising because among the Hausa/Fulani who, in this study constitute the highest proportion of respondents, female participation in labor force outside the home is not common. The traditional practice of confining married women to the house ("kulle") has restricted them to being "full time" housewives or homemakers.

The data Table 2 also indicate that $15.4 \%$ of the respondents' fathers and $62.2 \%$ of mothers married before their $20^{\text {th }}$ birthday. The percentage decreases as the age at marriage increases such that only $8.1 \%$ of mothers and $27.5 \%$ of fathers married at age 30 years and above. This means that slightly less than two third of mothers began marriage at their adolescent years, and almost three quarters of fathers began theirs during youthful years, before age 30 years. This pattern is expected in societies like Nigeria where marriage marks the point in a woman's life and childbearing is socially acceptable.

On fathers' type of marital union, the data indicate that $58.3 \%$ of the respondents' fathers were in monogamous union as against $41.7 \%$ in polygynous union. The high proportion of the respondents' fathers in polygynous relationship is explainable as the three states chosen for the study have a higher proportion of Moslems and Islam allows a man to take additional wives provided they would be treated equally. Besides, the absence of sexual satisfaction from the previous wife, her lack of capacity to produce children or required number/sex composition, sudden acquisition of wealth by a man, and the desire to form social and political alliance with strategically powerful patrons or godfathers, have also been found among the potent factors influencing polygyny in Nigerian society. 
Table 2: Percent distribution of respondents according to parental characteristics

Parental characteristics

All

$(\mathrm{N}=1175)$

Father's level of education

No formal schooling

$\%$

Some primary

26.3

Some secondary

15.9

Some tertiary

47.1

Mother's level of education

No formal education

46.2

Some primary

16.1

Some secondary

12.6

Some tertiary

Father's Occupation

Civil / public Servant

Business/Petty trading

28.1

Farming

18.8

Artisan

18.1

Unemployed

Mother's occupation

Civil/public servant

Business/petty trading

Farming

Artisan

Homemakers/unemployed

47.6

Father's age at first marriage

$<20$ years

20-29 years

$\geq 30$ years

Mother's age at first marriage

$<15$ years

$15-19$ years

43.3

20-29 years

29.7

$\geq 30$ years

Father's type of union

Monogamy

Polygyny

Father's total number of children

$\leq 6$ children

$\geq 7$ children

Mother's total number of children

$\leq 6$ children

$\geq 7$ children 
On the whole, there is the dominance of monogamy over polygyny in northwestern Nigeria, and this conforms to NDHS 2003 which found similar pattern (NPC, 2004). In essence, although polygyny was found to be dominant in studies on the African family system carried out in some selected societies (Assiemong, 1988), this is not entirely true of the Nigerian situation.

The distribution of respondents according to parental total number of living children is also indicated in Table 2. The data indicate that slightly more than one-third $(38.1 \%)$ of the respondents' fathers and largely more than two-thirds of their mothers, had a total of 6 living children or more. The average total number of living children for the respondents' fathers was 9.7 while that of the mothers was 5.9. Clearly, the fertility level of women is far less than that of men in northwestern Nigeria and is largely related to the polygynous nature of the area.

\section{Knowledge, Attitudes and Practice of Contraception}

In view of the apparent impact that knowledge and attitudes towards contraceptives have on their use, respondents were asked if they ever heard that there were methods used by married and unmarried persons to prevent, space or delay a pregnancy, and if they approve or disapprove using any of these methods. Questions relating to use of methods of contraception were also asked. Indeed data on knowledge and use of contraceptive methods are central to demographic analysis particularly fertility phenomenon. Knowledge about available contraceptive methods is the first step toward the adoption of appropriate methods. Table 3 presents responses on knowledge, approval and use of methods of contraception, by sex.

The data show that about $73 \%$ of male, and $65 \%$ of female adolescents have ever heard of methods of contraception, indicating that majority of adolescents of both sexes have some knowledge about contraceptive methods. The 2008 NDHS corroborates this high rate of knowledge of contraceptive methods in Nigeria. According to the NDHS report, $72.0 \%$ of all women age 15-49 years know at least one method of contraception/family planning and $71.0 \%$ know a modern method (NPC and ICF Macro, 2009:63).

However, males are more likely than females to be aware of these methods. While the proportion of female adolescents who approve contraceptive methods was higher $(33.4 \%)$ than their male counterparts (30.7), the proportion of both male and female adolescents who disapproved was clearly higher than those who approved. Slightly less than one-fifth of both male and female adolescents had ever used, while about one-sixth were currently using methods of contraception, suggesting that a large proportion of adolescents in northwestern Nigeria had never used and are not currently using methods of contraception. This upholds the observation that the total life situation of many teenagers in northwestern Nigeria tends to promote attitudes of fatalism which undermine the rational and planned acceptance or approval and use of contraceptive devices. 
Table 3: Percent distribution of responses to questions regarding knowledge, approval and use of methods of by sex

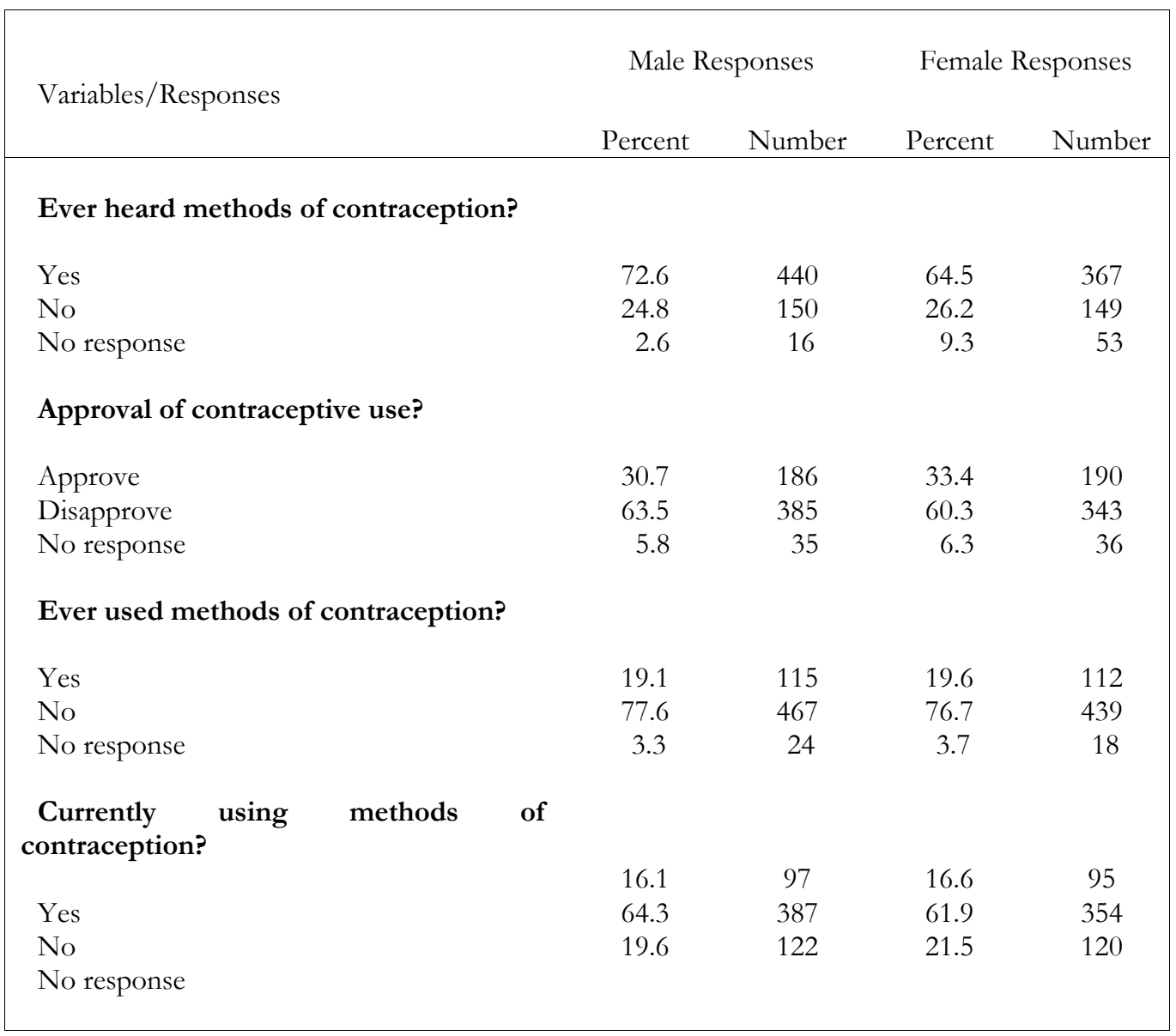

The relative gender imbalance in the level of awareness of methods of contraception among the respondents may not be unrelated with poor access to reproductive health information and low level of female education in the area. Generally in Nigeria many adolescents lack information on sexual health, the skills to negotiate sexual relationships, and access to reproductive health services. There is extreme lack of information or education for adolescents on sex, particularly teaching about sexuality and family planning. Among the Hausas in northern Nigeria, there is a widely-held belief that a girl should already be married and living in her husband's house at the time of the first menses so that she will be safely guarded in her husband's compound when she is able to conceive. Thus, young women may be denied access to sexuality or sex education as a means of discouraging them from sexual experimentation. This is also likely to have implications on their attitudes toward contraceptive methods which, as shown in Table 3 , were somewhat negative. 
Indeed, with regards to attitudes towards contraception probably the most important finding to emerge from the study is that knowledge and reported actual behavior have little in common. The findings show that knowledge of contraceptive methods is widespread; mention was made to some preventive methods to suggest that the fundamental barriers to behavioral change lie within the socio-cultural context that molds the reproductive behavior of the adolescents in northern Nigeria. Increasing obsession to dance to the tunes of religious tenets in this largely Islamic states combine with low level of education to support a culture in which marriage and childbearing begins early, particularly among female gender. A sense of invulnerability, adolescent sexual exploration and experimentation, entrenched reproductive norms and values, work together to maintain a situation that results in early childbearing and high fertility. Moreover, opportunities for young women in this setting to achieve an identity beyond those of wife and mother are nearly non-existent. In circumstances of entrenched power imbalances, adolescents' (particularly females) ability to dare into sensitive political and religious issue like contraception, or insist upon use of contraceptive methods, is low. There is need for intervention strategies to be undertaken to tackle issues relating to empowerment, and access to resources, improved information and services in northern Nigeria.

\section{Conclusion}

The findings have shown that social and economic circumstances of adolescents and their parents vary by gender. Therefore, acknowledging gender differences in the designs of programs for young people in the area may be a prerequisite for their success. Again, the findings of this study highlighted the immediate need for a concerted effort towards solving the several problems confronting the adolescents in northern Nigeria. At present, Nigerian Population Policy devotes little attention to the specific problems and needs of adolescents especially if they are unmarried. It is also evident that the society has a lot to gain from helping adolescents to make informed and responsible reproductive choices. By and large, this broad based description of the sociodemographic characteristics of the sampled adolescents is intended to stimulate discussions among policy makers, programmes planners, and researchers on what we know about adolescents in northern Nigeria and to assess whether current resource allocations and programmes are appropriate for the needs of this large segment of the population.

\section{References}

Adeokun, L. A. (1979). "Family Planning in Southwestern Nigeria: Levels and determinants of KAP." 1971-75 National Survey of Fertility, Family, and Family Planning, Phase I:

S. $\quad$ W. Nigeria. DSS Monograph No.1. University of Ife, Ile-Ife. Pp.62-104.

Assiemong, M. (1988) "Religious Mores and norms, and Family Building in Africa". RIPS Occasional Paper, Vol.1, No.1, Pp 44-61

Bledsoe, C. and B. Cohen (eds) (1993) Social Dynamics of Adolescent Fertility in sub- Saharan Africa. Washington, DC: National Academy Press. 
International Labour Organization (ILO) (1986). Economically Active Population: $\quad$ Estimates and Projections 1950-2025 Vol.iv: Methodological Supplement. Geneva: International Labour Office

International Planned Parenthood Federation (IPPF) (1994) Understanding Adolescents. London National Population Commission (NPC) [Nigeria] and ICF Macro (2009). Nigeria Demographic and Health Survey 2008. Abuja, Nigeria: National Population Commission and ICF Macro.

- and ORC Macro (2004). Nigeria Demographic and Health Survey 2003. C Calverton, Maryland: National Population Commission and ORC Macro.

- (2002). Children, Adolescents and Youth. Abuja: National Population Commission.

. (2002). Sentinel Survey of the National Population Program, Baseline Report 2000. Abuja: National Population Commission.

Population Reference Bureau. (2005) Women of Our World. Washington, DC.

. (1992). Adolescent Women in sub-Saharan Africa.A Chart-book on marriage and childbearing. Washington, DC.

United Nations. (1989). Adolescent Reproductive Behavior: Evidence from Developing Countries,

Vol.2. New York: United Nations.

. (1988a). Adolescent Reproductive Behavior. Evidence from Developed Countries.

ST/ESA/SER.A/109. New York.

. (1987) Fertility Behavior in the Context of Development: Evidence from the World Fertility Survey. Population Studies No. 100. ST/ESA/SER. A/100. New York.

Vandemoortele, J. (1991). "Labour Market Information in Sub-Saharan Africa" in G. Standing and V. Tokoman (eds.) Towards Social Adjustment: Labour Market Issues in Structural Adjustment. Geneva: International Labour Office. Pp. 81-113.

Wall, L. L. (1998) "The social context of Maternal Morbidity and Mortality among the Hausa of Northern Nigeria". Studies in Family Planning vol. 29, No. 24.

Web, W. (1994). Teens Sexuality: Empowering Teens to Decide. Policy studies Review 13 Vol. 29, No.2

World Health Organization (1989a). The Health of Youth. Background Document, Technical

Discussions 1989. A42/Techincal Discussions/2. Geneva.

. (1989b). The Health of Youth. Facts for Action: Youth and Reproductive Health. A42/Technical Discussions/5. Geneva.

Yinger, N.; A, de Sherbinin; L. H. Ochoa; L. Morris, and J. Hirsch (1992) Adolescent Sexual Activity and Childbearing in Latin America and the Carribbean: Risks and Consequences. Washington, DC: Population Reference Bureau. 